\title{
A phenomenological inquiry into the lived experience of Adults diagnosed With Attention Deficit Hyperactivity Disorder (ADHD) Employed by the NHS.
}

\begin{tabular}{|r|l|}
\hline Journal: & Mental Health and Social Inclusion \\
\hline Manuscript ID & MHSI-11-2020-0075 \\
\hline Manuscript Type: & Primary Research Paper \\
\hline Keywords: & ADHD, Adult, Employment, Lived Experience, Qualitative, Management \\
\hline
\end{tabular}


'AWArE': NHS Employees with ADHD: The lived experience

\begin{abstract}
Introduction: Approximately $3.5 \%$ of the workforce is diagnosed with Attention Deficit Hyperactivity Disorder (ADHD). With a greater risk of work-related stress and absences, workbased social interactions and environments are significant factors. Nurses experience better occupational outcomes than other professions, indicating that further investigation is important to understand why healthcare roles can better sustain employees with ADHD.
\end{abstract}

Method: Interpretative phenomenological analysis (IPA) was used to explore how employees with ADHD both experience and make sense of their employment and professional relationships. Participants self-identified from two NHS Trusts and participated in semi-structured interviews.

Findings: 7 interviews contributed to the final analysis. 4 themes emerged; Social Environment (Relationships), Physical Environment, Behaviour and Work Tasks. Complex interactions were observed between individuals' internal constructs and overall employment experience.

Conclusion: Dynamic and interactive components affect employee volition and performance. The NHS provides variety, flexibility and encourages self-motivation suiting employees with ADHD and with adequate support enables enjoyable, successful NHS careers.

Originality: This is a novel study that explores the psychodynamic and physical presentations of ADHD providing a unique perspective on the challenges and strengths off employees with ADHD Practical implication: The findings can be used to inform local NHS management frameworks for supporting workforce with ADHD

Keywords: Adult, ADHD, Employment, Lived Experience, Qualitative, Management 


\section{Introduction}

Employment strongly influences a range of needs endorsing personal value and societal contribution (Bryant et al (ed) 2014). The work environment, an arena for complex personal and social interactions (Adamou et al, 2013), creates both positive and negative demands on an individual (Parkinson S et al, 2004). Successful navigation of the work environment is implicit for successful occupational and social performance (Parkinson S et al, 2004).

Approximately $3.5 \%$ of the workforce (De Graaf et al 2008) has received a diagnosis of Attention Deficit Hyperactivity Disorder (ADHD) a lifelong neuropsychological disorder characterized by distractibility, hyperactivity and impulsiveness (Adamou et al, 2013; Matheson et al, 2013). Traits such as restlessness, disorganisation, being over-whelmed, and procrastination (Adamou et al, 2013; Carr M, 2012; Grossberg 2005; Hall et al 2013 and Matheson et al, 2013) all contribute to feelings of failure and shame (Painter, 2008 and Matheson et al, 2013).

\section{ADHD in the workplace}

Work relationships are integral to effective occupational engagement and volition (Willerton, 2010). The perceived quality of these relationships is influenced by a myriad of internal and external experiences, however ADHD traits can significantly impact on this experience resulting in frequent employment changes (Hall et al 2013), lower wages (Biederman \& Faraone, 2006), fewer promotions and more dismissals (Painter et al 2008, Murphy \& Schachar, 2000). Furthermore, higher susceptibility to stress, burnout and absenteeism (Brattberg 2006) cause significant life-long financial burdens (Adamou et al, 2013).

Literature reveals a paucity of research exploring the lived experiences of working adults with ADHD. Whilst most papers present a negative account, Grossberg (2005) highlighted an underrepresented perspective where ADHD brings significant strengths. Halbesleben (2013) also presented an interesting exception to the negative trend where nurses with ADHD experienced longer employment tenure (mean 12.4yrs), compared to people with ADHD not working in 
healthcare (mean 6.41yrs). This indicates potential unexplored factors within a healthcare environment or culture that engage this population better than other working environments warranting further investigation.

This study aimed to understand how key traits of ADHD help or hinder overall work experience within the healthcare setting, and identify strategies to support occupational performance and wellbeing through qualitative interviews with healthcare staff living and working with ADHD.

\section{Methodology}

Approval was obtained from the Health Research Authority to conduct the research. Interpretative phenomenological analysis (IPA) was chosen as it gains a greater depth of understanding by reflecting on both the individuals' and researcher's perceptions and insights (Smith J \& Osborn M, 2008) on their working and wider world and requires a small sample size (Smith \& Osborn 2011) due to the intricate analysis of the data.

The study recruited from two NHS Trusts (Acute and Mental Health) through a convenience sampling method. All participants self-referred After responding to study promotion via NHS Trust communication systems, posters and clinical services.

Inclusion criteria:

- $16+$ years with primary diagnosis of ADHD.

- Paid employment within NHS organisations or agencies that provide NHS cover.

- Fluent in English.

\section{Exclusions:}

- Healthcare staff in training/placement.

\section{Data collection:}


Both the primary researchers are NHS employees with MScs in clinical research. Supervision was sought from an academic lecturer experienced in IPA. An NHS employee with lived experience of ADHD and independent from the study supported the team verifying relevance and accuracy.

All practices adhered to the required legislation and approvals and was adherent to the Declaration of Helsinki and General Data Protection Regulations.

Potential participants were provided with a participant information sheet a minimum of a week prior to the interview and provided informed consent following the approved process.

Semi-structured interviews were conducted using 'funnelled' interview schedules to guide the discussion (Smith \& Osborn 2011). 'Funnelling' initially explores broad topics then draws the focus to the more specific areas of inquiry. Interviews were recorded and transcribed by the interviewer as part of the reflective process.

Pseudonymised codes were assigned and identifiable information was removed during transcription.

Analysis broadly followed Colaizzi stages (1978) as outlined in Wojnar DM \& Swanson KM (2007).

Codes were drawn from the transcripts alongside using the primary researchers interpretative reflections, adhering to the IPA's 'double hermeneutic approach' (Smith \& Osborn, 2008).

These codes were clustered into themes and organised using the Model of Human Occupation (MOHO) framework (Parkinson S et al 2004) enabling interpretation of the complex and interwoven impacts of the ADHD, the employee and the work environment.

\section{$\underline{\text { Results }}$}


7 participants were interviewed. The interviews ranged between 23 - 66 minutes.

Some demographic information was gathered and presented in Table 1: 


\section{Table 1: Demographics of participants (NK= not known)}

\begin{tabular}{|c|c|c|c|c|c|c|c|c|c|c|c|c|c|c|c|c|c|c|c|c|c|c|c|}
\hline 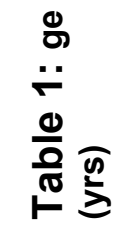 & & c) & & 옹 & & 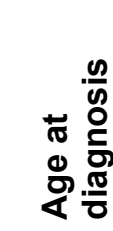 & & 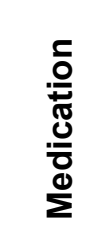 & & 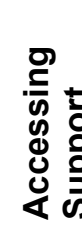 & & $\begin{array}{l}\stackrel{0}{2} \\
\stackrel{2}{1} \\
\stackrel{0}{0}\end{array}$ & & $\begin{array}{l}0 \\
\frac{4}{4} \\
\frac{0}{0} \\
\frac{0}{0} \\
0\end{array}$ & & 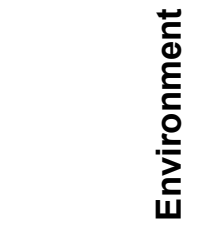 & & $\begin{array}{l}\frac{0}{5} \\
\frac{1}{0} \\
\frac{0}{6} \\
\stackrel{0}{0}\end{array}$ & & $\begin{array}{l}\frac{0}{5} \\
\frac{c}{0} \\
\stackrel{1}{上} \\
\frac{0}{1} \\
\frac{2}{z}\end{array}$ & $\frac{n}{2}$ & $\begin{array}{l}\frac{n}{2} \\
\stackrel{n}{2} \\
\frac{n}{1} \\
\frac{1}{Z}\end{array}$ & \\
\hline $20-29$ & 1 & Male & 3 & Hyperactive & 0 & $<20$ & 1 & Yes & 5 & Yes & 4 & Clinical & 5 & $1-4$ & 3 & Community & 3 & $0-5$ & 6 & $<1$ & 2 & $\begin{array}{l}\text { Mental } \\
\text { Health }\end{array}$ & 6 \\
\hline $30-39$ & 1 & Female & 4 & Inattentive & 2 & $20-29$ & 1 & No & 2 & No & 3 & Other & 2 & $5-7$ & 3 & Ward & 2 & $6-10$ & 1 & $1-5$ & 1 & Acute & 1 \\
\hline $40-49$ & 2 & & & Combined & 5 & $30-39$ & 2 & & & & & & & NK & 1 & Office & 1 & & & $6-10$ & 2 & & \\
\hline $50-60$ & 2 & & & & & $40-49$ & 3 & & & & & & & & & Other & 1 & & & $11+$ & 2 & & \\
\hline NK & 1 & & & & & & & & & & & & & & & & & & & & & & \\
\hline
\end{tabular}




\section{Observations:}

The majority of participants (5/7) were over the age of 30yrs when diagnosed with ADHD. All participants worked directly with patients.

Most participants sought assessment following recommendations from others, (P2) "Some friend's...father worked in psychiatry and...thought I was displaying a lot of the signs and sent me for a diagnosis. At that point I still thought [ADHD was] slightly made up if I'm honest I was part of that group of people." intimating that prior to receiving their diagnosis participants did not attribute their behaviours to ADHD, and initially questioned the legitimacy of the disorder before accepting and understanding the diagnosis in context of their behaviours.

(P1) "This is part of me it doesn't mean I am a lazy waster...I found it quite liberating...I didn't have to try and make excuses." 
Analysis relating to the lived experience of ADHD in the working environment identified four main themes as depicted in Figure 1:

\section{Figure 1: Emergent themes from the data analysis}

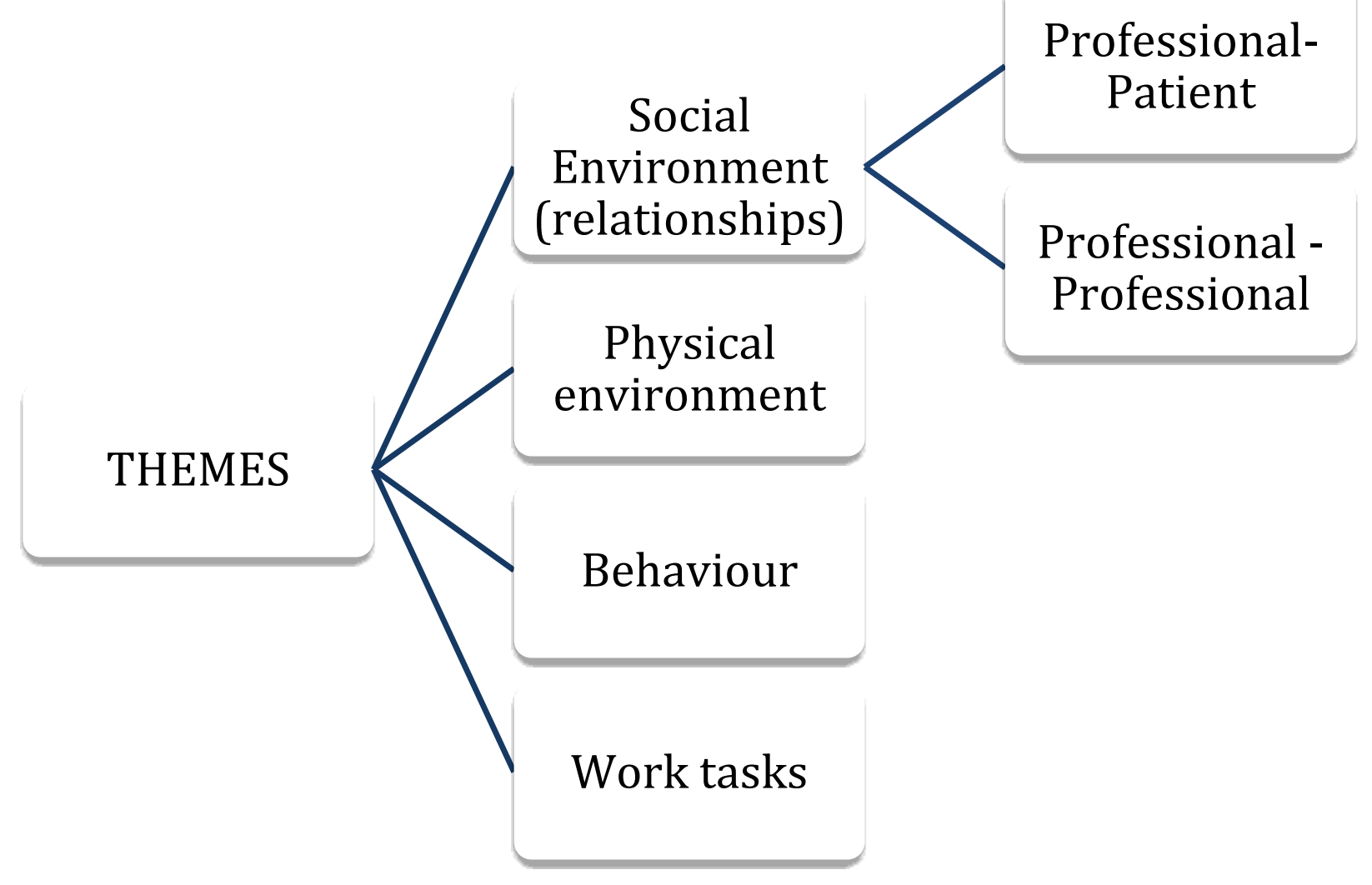

\section{Theme 1: Social environment (relationships)}

Two types of relationships impacted by ADHD emerged as 'professional - professional' and 'professional - patient'.

Participants appeared to attribute a stronger sense of personal causation and satisfaction to their 'professional-patient' relationships whilst the 'professional-professional' relationship was interpreted as more challenging and imbalanced, resulting in them feeling more exposed and disempowered.

\section{Professional - professional}


The nature of professional-professional dyads was observed to be strongly influential on an employee's sense of confidence and identity within the team. Predetermined negative selfassessments contributed to their perspectives of these relationships.

(P7) "I just had myself down as a scatterbrain."

(P8) "I have a low opinion of myself."

Whilst, assumptions that those colleagues viewed them as being "troublemakers" (P5), "uncaring" (P6), "uneducated" (P6) or just "reckless" (6) along with preconceptions of ADHD related behaviour impacted their sense of identity within the team:

(P8) "People just assume ...you're bouncing around walls."

Further assumptions surrounding colleagues' attitudes contributed towards a 'child-parent' dynamic rather than nurturing a sense of equality further generating feelings of difference, isolation and invisibility. Participants expressed beliefs around reprisal "Will they send me to the special room?" (P2) inducing behaviours that seek acceptance. "I will really try and behave." (P4)

This dynamic led to a strong shared belief of being misunderstood, not just in terms of the traits but also consequences for each participant,

"They don't realise how much of an effect... ADHD can have on your life...there's not much understanding for us." (P6)

The sense of acceptance and capability was strongly influenced by either the praise or criticism received by colleagues. Both inducing strong emotional reactions with participants referring the experiences as, being like "catnip" (P7) or they would "internally crumble" (P2). Feelings of low self-esteem; "not being good enough" (P2), "blagging" (P7) your career whilst "deceiving" (P7)all around about one's capability or a polarized experience of elation would dominate the sense of 'l', creating an inner turmoil that would go unnoticed by colleagues.

(P8) "A lot of your life ...feeling...less a person...the knock - on effects are quite...devastating." 
(P7) “I often felt that l've been blagging it my entire career ... deceiving people because I struggled to do certain things [however] If I'm ... praised it's like catnip"

Managers' responses to an employee's ADHD disclosure was critical. Negative attitudes reduced trust between employee and manager with the participant disclosing feelings of being dismissed or disbelieved.

(P8) “Disclosing didn't work out very well...because l've got ADHD she [Manager]...tutted.”

Whereby, managers who engendered acceptance and understanding, promoted a sense of security. These affirming relationships further influenced positive identity by promoting confidence, capability and effectiveness reinforcing positive daily experiences, a sense of value and successful practice. Positive workplace cultures were considered pivotal in reducing anxiety. Empowering and enabling the participants improved productivity and engagement in a way that embodied their values of professionalism and integrity;

(P6) "My managers allowed me to be my own worker... enabled me to be at my most efficient." (P6) "Going through a few jobs until you find someone or a manager that truly understands, then it's worth it...Succeed because of ADHD not in spite of it."

It was important for the participants to be viewed as competent and valued members of the team calling for their colleagues to "realise [they] can be as successful as anybody else" (P8). When they felt treated like a "normal person" (P2) with their diagnosis "not being an issue" (P2) it could be viewed as a potential asset. Participants described being able to "do lots of good stuff with it, be a positive influence" (P7). This would lead to feelings of being empowered and the role be one that is "rewarding" (P8). A strong sense of loyalty and commitment was emphasised that went with collaborative and trusting work- based relationships 
(P6) "People think we are not reliable but given the right position and...support we are probably the most loyal and reliable people...we...try and put our all in everything ...complacency, we really don't have that kind of personality."

\section{Professional - client}

All participants identified personal values that they felt helped built co-operative and person centred relationships with their patients. Aspects such as altruism, professional integrity, passion and a genuine regard appeared to be fundamental traits they brought to the 'professional - client' relationship suggesting clear and established purpose and empowerment within this role. The confidence that participants felt in their clinical skills and ability to build a therapeutic relationship with their clients suggest a positive framing of living with ADHD within the healthcare workplace, grounded within their compassionate values and commitment to others. This was in sharp contrast to professional relationships with peers, colleagues or managers which felt less empowering and ultimately led to reduced self-esteem.

(P4) "I am genuinely interested in the patients."

(P6) "Knowing that l'm making a difference...I need something that means something to me.

\section{Theme 2: Physical Environment}

Health care environments are often busy dynamic settings with a myriad of sensory stimuli, "Lots of noise, colour, bright lights, people" (P1) making distractions a common consequence being the difficulty to focus. "people constantly walking up and down... I couldn't concentrate." (P8). For the employee with ADHD the inability to filter external stimuli creates a significant daily battle:

Sensitivity to noise is particularly prevalent with the slightest background noises becoming sources of overwhelming irritation and distress "People eating lunch out of plastic boxes, making 
that noise...drives me CRAckers...I am completely absorbed" (P4), whilst multiple conversations demands the employees' full attention with significant consequences "I want to be involved in them all...I will latch onto any sound,... I get overwhelmed very easily.” (P6). The descriptions such as "absorbed" (P4) and "latch" (P6) giving us the indication of the draw that everyday background noise demands.

(P8) "Every time she touched her calculator I lost concentration."

This overwhelming sensory experience alongside the excitable ADHD mind lures the employee away from their task in hand. Individuals have found ways to manage their distractibility, sometimes necessitating isolating away from their colleagues, even though this brings its own anxieties.

(P1) "People used to think I was... being an antisocial miserable git ... I just couldn't cope with the noise."

Despite these challenges, participants appear to struggle to acknowledge a need for support appearing, at times, conflicted and incongruent about the provision of workplace adaptations;

(P4) "I would hate to feel I was being accommodated because of my diagnosis ..."

[...]"...perfectly reasonable that provision might be made...the Trust could be mindful of the things that cause people's inattention to be worse."

(P1) "The message is that we are different, we're not worse... I suppose some people are better... just need a little bit of extra help".

Some participants express that self-management and care for their condition and adaptations lay with them alone, with a sense that it was their responsibility to 'master' their condition in order to succeed in the workplace.

(P5) "I have to change myself to fit in with work rather than changing to fit in with me"

(P7) "What I've got to do is work with the environment that l've got." 


\begin{abstract}
This complex dynamic between the external and internal factors often leaves employees feeling unsettled and dissatisfied, where their experiences of living and working with ADHD in less supportive environments led to a feeling of needing to constantly move on and, ultimately, contributing to an unstable job history.

(P1) "I felt fractured...escaping jobs rather than going to jobs."

(P7) "There's something a little depressing about the fact that l've run out of places to go."
\end{abstract}

\title{
Theme 3: Behaviour (Traits)
}

Behaviour impacts on our ability to organise performance within an environment (Kielhofner $G$ 2008) such as the workplace. A constant presence, ADHD traits directly influence behaviours bringing tangible cognitive, psychological and physical experiences. Participants shared phenomena such as a sense of a "motor running" (P5) or that their minds being busy "24/7" (P6) going a "million miles an hour" (P5) giving a sense of cognitive and sensory hyperactivity, leading to feelings of being "irritable" (P4) and "impatient" (P4) There were more individual experiences that were equally distressing

(P1) "I get a feeling sometime, I refer to it as spiders in my head, it feels like little things are crawling all over my head and it's really uncomfortable."

These traits, along with a predominant anxiety specifically impaired engagement. The propensity to "over analyse, ruminate all the time" (P7) prevents the ability to prioritise and organising effectively along with a sense of being "frozen with anxiety" (P2) that impacts on the ability to "motivate and initiate" (P2) activities.

Some behaviours were externalised via motor and impulsive actions with participants describing how they could "randomly jump up" (P8) or find themselves "bouncing" in their chair (P4). These behaviours were difficult to "regulate" (P8) leaving them tired and "exhausted" (P8) from the 
persistent effort used in endlessly trying to "master" (P4) these behaviours, navigate the consequences and adapt to perform effectually.

(P8) "Very difficult to regulate myself... it's really tiring...sometimes I just feel exhausted." (P6) "I've made appalling decisions based on... my impulse...took me a long time to manage how to listen... take it all on board without becoming distracted."

Dichotomously some characteristics were considered niche skills such as being visionaries, creative "outside the box" (P8) thinkers with "The ability to think in lots of different way in one go" (P5) where “, ideas just present themselves.” (P5) as well as being more risk aware being "more aware of dangers" (P6). When engaged and focussed participants felt that they were specifically efficient "I can do the work as fast as anyone...I am hyper organized." (P4)

This polaric and changeable nature of ADHD, with the propensity to swing between efficient and organised, to unproductive and inattentive elicited confusion with participants reporting that they placed pressure on themselves to consistently perform well. A negative internal dialogue let to self-negating thought patterns such as believing themselves to be "useless" (P8) or "all the promise they saw, I wasn't able to deliver" (P7). This would lead to a lack of confidence in abilities and a reluctance to applying for higher banded roles (P7) "I'm wary about moving up and exposing myself."

\section{Theme 4: Work Tasks}

Clinical roles are responsive, changeable and varied and can be both sedentary and active. As the ADHD brain is "Wired for adventured...constantly looking for excitement!" (P4) tasks must engage an individual quickly and maintain interest; “"if things don't interest me I don't notice” (P7), 
potentially leading to misunderstanding around professional integrity (P7) elaborating further that "it's not that I am not deciding not to do them, l'm not noticing."

The variation and changeability enables a person with ADHD to remain interested and be responsive to any given situation (P4) "I love the fact that no days ever the same ...It is constantly stimulating... we have to think quickly." Whereas the more sedentary roles such computer work and long meetings hold lower satisfaction value and are experienced as; "menial" (P6) and "mind-numbing" (P1)

These perceived mundane tasks reduced productivity and exacerbated impulsive behaviours; "If somebody's talking to me... and I get bored I find, I'm up out of my chair and walking away." (P4)

"I become restless...they might think l'm being rude and it's really hard for me to sit there." (P8)

Maintaining attention proved difficult where both internal and external stimulation further create distraction, being drawn in by what is "exciting and stimulating" (P4) and at times demanding immediate reactions, with the effort to return to a task and remain focussed being "100 times harder" (P8). Productivity and capacity become hampered as they are drawn in by and unable to filter out the superfluous distractions, the leading the participant to become overwhelmed, unfocussed and frozen.

(P2) "Brain gets so scrambled as to make me...less useful."

(P5) "It all gets too overwhelming and I have to lock myself in the toilet for 5 minutes."

\section{Coping Strategies}

In order to manage this myriad of challenges, perform efficiently and maintain professional integrity, the employee with ADHD utilised a variety of pharmacological, organisational, cognitive, and physical coping strategies. 
The majority of participants took medication (5/7), with positive effects on concentration "Organise thoughts, focus a little better" (P7), self-esteem "feeling less bad about myself" (P4) and anxiety levels "I almost feel completely relaxed" (P7). Being able to negotiate a suitable regime with their doctor helped ensure maximum benefits for their work environment.

(P4) "Ritalin definitely changed things, ...When I do nights it's really difficult to know when to take it, we've found a way round it."

Organisationally, participants found having autonomy to organise their own timetable and work independently enabled them to manage caseloads more successfully "I tend to see people more often than my colleagues." (P6). Utilising strategies to aid memory "I have little prompts" (P8) and also grounding, mindfulness techniques, to help challenge negative thinking patterns "bringing myself back to the facts of life...reel myself in." (P2), "taught my-self to be in the present." (P6) all served to improved positive and effective engagement within the workplace.

\section{Discussion and Implications}

Coming to terms with a diagnosis of ADHD during adulthood and navigating the various life domains such as the workplace, inferred a journey that necessitated challenging beliefs and ongoing adaptation, a process reported by Young S et al (2008).

Successful adaptation is dependent on any number of intrinsic and extrinsic factors. The workplace brings specific challenges and opportunities that influences occupational performance and resilience.

Relationships had the greatest overarching effect on occupational identity, participation and performance. It was of particular interest that participants implied a greater confidence in their relationships with patients as opposed to their colleagues and that being 'front line' contributed 
strongly to their sense of fulfilment. Employees with ADHD tended to experience low self-esteem reinforced by cognitive distortions and historical experiences, placing them at a disadvantage in their perceptions of the professional-professional balance. Personal causation and perceived clinical effectiveness were powerfully related to the quality of external validation received.

Participants displayed a continuing conscious effort to manage the adverse thought processes, perceptions and anxieties guarding against the ongoing negative cycle. They become vigilant to colleague's perceptions not wanting to let their co-workers down or feel that their ADHD defined them. This effort rendered even the most meaningful interactions potentially problematic. This finding supports findings by Frederiksen et al (2014) who examined ADHD within long-term workplace disability and attributed high levels of clinical anxiety to significant occupational impairment, particularly in female workers.

Extending from this the balance of the physical environment was integral in an employee's ability to engage in relationships and work tasks. Busy environments created high levels of distraction. Whilst noise was specifically diverting, it was its all-consuming quality that was particularly interesting, with the quietest of noises commanding absolute attention. Multiple distractions could not be filtered out, competing for priority, creating an overwhelming demand that could be distressing and necessitating the need to withdraw. This may cause a conflict in certain healthcare work environments, where the balance between feeling most valued on the clinical frontline, while needing a quiet space to concentrate may not be possible in busy patient areas.

Behaviours associated with ADHD governed both environmental and relationship experiences. Originating from impulsive and habituated internal physical, cognitive and emotional drives these behaviours can manifest as strong kinaesthetic experiences such as "crawling spiders" (P1) or "revving motors" (P5) lurking in the conscious awareness. Described as something to be mastered and "reigned in"(P4) we get a glimpse of the tough and relentless reality of the effort 
required to sustain effective performance when living with ADHD and working. The healthcare setting seems to allay some of the challenges through its varying nature, and flexibility.

Behaviours experienced as hindering served to reduce self-confidence and self-esteem whilst those behaviours considered enabling and unique, coupled with positive validation from colleagues and management, facilitated adaptation, engagement and performance particularly when working with healthcare clients. This is in line with findings from Sarkis et al (2014) who emphasises the positive contribution that creative ADHD traits can make to the healthcare workplace. The areas of positivity defined by our participants are similar to those presented by Sedgwick et al (2019) whose work emphasised the cognitive dynamism and energy of people with ADHD. However, there was also internal conflict observed between an employee's need for workplace adjustments and their wish to be treated and viewed as 'normal'. Whilst employees acknowledged responsibility for their ADHD greater understanding, support and flexibility from managers was deemed vital in enabling a more fulfilling and valued work experience.

Work tasks varied greatly creating diverse reactions between those perceived as 'meaningful' and 'menial.' Time was automatically apportioned to 'hands on', varied and responsive tasks however it was imperative that a sensitive balance was maintained between these compelling roles that engender motivation, focus and skill from becoming overloaded, overwhelmed and pressured reinforcing the adverse effects of high self-criticism.

The self-motivated ethos of healthcare organisations engaged practices that value independence and flexibility. This, along with a value base that engenders high levels of empathy for both colleagues and service users, creates a culture that is often supportive, and utilises specific skill characteristics of conditions like ADHD. These narratives validate the observation that Nurses with ADHD had longer tenures than their employed colleagues outside of healthcare setting. 
Current research highlights a gap between employment potential and attainment for those with ADHD. This research provides deeper understanding of causality by identifying specific challenges to employment based occupational participation, personal effectiveness and identifying the skills and strengths of this population, demonstrating that ADHD alone is not a barrier to success. It also suggests that a greater understanding, and positive working relationships are the important areas for development where this is lacking.

\section{Recommendations}

This study has demonstrated the need for collaborative working, increased awareness of ADHD and support on an organisational, managerial and peer level.

- Organisationally and locally promoting a positive work culture that embraces the strengths and qualities of this population whilst exploring adaptations that are beneficial to all employees as well as those with ADHD.

- Managers need to positively negotiate and promote flexible work practices that target strengths and minimises challenges by agreeing action plans to help navigate specific challenges.

- Further research in other work-based environments to compare and contrast findings in this paper to fully support policy and service development including employment skills training

\section{Limitations}

The study mainly recruited employees from a single mental health trust leading the findings to be more specific to that environment. Participants were limited to those who were maintaining professional positions in the workplace, and therefore the views and experiences of people who had left the healthcare environment due to their ADHD were not examined. 


\section{What the study has added:}

An intimate understanding of the experiences of employees with ADHD providing depth to current literature which empowers colleagues to build more positive and effective workplace relationships. 


\section{$\underline{\text { References }}$}

Adamou M, Arif M, Asherson P, Aw T, Bolea B, Coghill D, Guojonsson G, Halmoy A, Hodgkins P, Muler U, Pitts M, Trakoli A, Williams N, and Young S (2013) Occupational issues of adults with ADHD. BioMed Central psychiatry 13(59): 1-7.

Bierderman J and Faroane S (2006) The Effects of Attention-Deficit/Hyperactivity Disorder on Employment and Household Income. Medgenmed.2006; 8(3): 12

Brattberg G (2006) PTSD and ADHD: underlying factors in many cases of burnout. Stress and Health 22: 305-313.

Bryant,W, Fieldhouse J, and Banningan K (2014) Creek's Occupational Therapy and Mental Health. London: Elsevier Health Sciences UK.

Carr M (2012) ADHD and anxiety in adults. e- book 14\% Amazon Kindle.

De Graaf R, Kessler RC, Fayyad J, ten Have M, Alonso J, Angermeyer M, Borges G, Demyttenaere K, Gasquet I, de Girolamo G, Haro JM, Jin R, Karam EG, Ormel J, Posada-Villa J (2008) The prevalence and effects of adult attention-deficit/hyperactivity disorder, (ADHD) on the performance of workers: results from the WHO World Mental Health Survey Initiative. Occupational and Environmental Medicine 65(12): 835-842.

Fredriksen M, Dahl AA, Martinsen EW, Klungsoyr O, Faraone SV, Peleikis DE. Childhood and persistent ADHD symptoms associated with educational failure and long-term occupational disability in adult ADHD. Atten Defic Hyperact Disord. 2014 Jun;6(2):87-99. doi: 10.1007/s12402014-0126-1.

Grossberg B: (2005) Making ADD work. Perigree: The Berkley Publishing group.

Halbesleben J, Wheeler A and Shanine K (2013) The Moderating Role of AttentionDeficit/Hyperactivity Disorder in the Work Engagement-Performance Process. Journal of Occupation Health Psychology 18(2): 132-143.

Hall C, Newell K, Taylor J, Sayal K, Swift K and Hollis C (2013) Mind the gap - mapping services for young people with ADHD transitioning tom child to adult mental health services. BioMed Central Psychiatry 12:186. 
Matheson L, Asherson P, Wong I, Hodgkins P, Setyawan J, Sasane R and Clifford S (2013) Adult ADHD patient experiences of impairment, service provision and clinical management in England: a qualitative study. BioMed Central Health services research 13: 184.

Murphy P and Schachar R (2000) Use of self-ratings in the assessment of symptoms of Attention Deficit Hyperactivity Disorder in Adults. The American Journal of Psychiatry 157(7): 1156-1159.

Painter C, Prevatt F and Welles T (2008) Career beliefs and job satisfaction in adult with symptoms of attention deficit/hyperactivity disorder. Journal of employment counselling 45(4): 178-188.

Parkinson S, Forsyth K, and Kielhofner G (2004) The user's manual for Model Of Human Occupational Tool (MOHOST). Available at: http://moho.uic.edu/pdf/MohostManual.pdf (accessed 02 May 2019).

Sarkis E. Addressing attention-deficit/hyperactivity disorder in the workplace. Postgrad Med. 2014 Sep;126(5):25-30. doi: 10.3810/pgm.2014.09.2797. PMID: 25295647

Sedgwick JA, Merwood A, Asherson P. The positive aspects of attention deficit hyperactivity disorder: a qualitative investigation of successful adults with ADHD. Atten Defic Hyperact Disord. 2019 Sep;11(3):241-253. doi: 10.1007/s12402-018-0277-6

Smith J and Osborn M (2008) Qualitative Psychology: A Practical Guide to Research Methods. 2nd edn. London: SAGE Publications Ltd.

Willerton J (2010) Psychology of relationships. New York: Palgrave Macmillan.

Young S, Bramham J, Gray K, and Rose E (2008) The Experience of Receiving a Diagnosis and Treatment of ADHD in Adulthood. Journal of Attention Disorder 11(4): 493-503 
Figure 1: Emergent themes from the data analysis

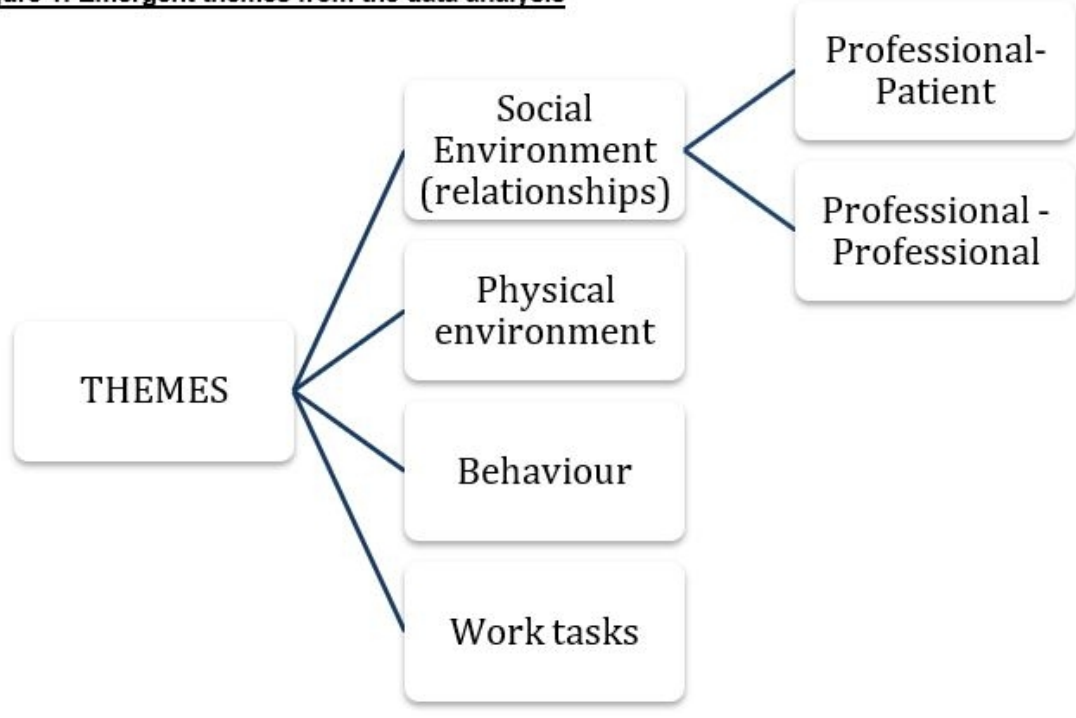

Figure 1: Emergent themes from the data analysis

$159 \times 91 \mathrm{~mm}(120 \times 120 \mathrm{DPI})$ 